\title{
Estresse e relações familiares na perspectiva de irmãos de indivíduos com Transtornos Globais do Desenvolvimento ${ }^{1}$
}

\author{
Vanessa Fonseca Gomes \\ Cleonice Bosa \\ Universidade Federal do Rio Grande do Sul
}

\begin{abstract}
Resumo
Os Transtornos Globais do Desenvolvimento (TGD) caracterizam-se pelo comprometimento da interação social, pelo uso estereotipado e repetitivo da linguagem e pela presença de comportamentos repetitivos com interesses restritos. A maioria dos estudos nessa área focaliza o impacto dos TGD nos pais, ao passo que pouca atenção tem sido dada ao impacto nos irmãos. O presente estudo objetivou investigar a presença de indicadores de estresse e a qualidade das relações familiares em irmãos de indivíduos com e sem TGD. Participaram 62 crianças e adolescentes com idades entre 8 e 18 anos. Os resultados revelaram a ausência de indicadores de estresse nos grupos investigados. Os dados corroboram as premissas do metamodelo de Adaptação da Família à Doença Crônica, ao demonstrar que a presença de um membro com TGD na família não representa, obrigatoriamente, um evento adverso para os irmãos, desde que haja qualidade nas relações familiares e uma rede de apoio.
\end{abstract}

Palavras-chave: transtornos globais do desenvolvimento; autismo; relações familiares; estresse; irmãos

\begin{abstract}
Stress and family relationships from the perspective of siblings of individuals with Pervasive Developmental Disorders. The Pervasive Developmental Disorders (PDD) can be described as a severe developmental disorder that presents deficits in reciprocal social interaction, communication and restricted and repetitive interests and behaviors. Most studies focus the impact of parenting an individual with PDD. However less research has investigated how autistic individuals affect their siblings. The goal of this study was to evaluate indicators of stress and the quality of family interactions in siblings of autistic individuals compared to siblings of normally developing individuals. Sixty-two siblings between the ages of 8 and 18 participated in this investigation. Results indicated that siblings of individuals with autism, as well as comparison siblings, do not report stress. The results of this investigation suggest that the presence of a family member with PDD does not necessarily represent an adverse event for the siblings. This could be due to the quality of family relations and availability of social support.
\end{abstract}

Keywords: pervasive developmental disorders; autism; family relations; stress; siblings

$\mathrm{O}$ presente estudo tem como objetivo principal investigar a presença de indicadores de estresse e a qualidade das relações familiares em irmãos de indivíduos com Transtornos Globais do Desenvolvimento (TGD). Para tanto, inicialmente se apresenta o conceito de estresse. Em seguida, aborda-se a questão da definição de Transtornos Globais do Desenvolvimento (TGD) e sua relação com o estresse familiar, sendo o estresse em irmãos de crianças com TGD, particularmente focalizado. O modelo biopsicossocial (Bradford, 1997) é utilizado na compreensão do fenômeno investigado.

\section{Estresse}

O estresse é compreendido pela maioria das pessoas como uma forma extrema de emoção. O medo, a raiva, a tristeza e até mesmo a alegria, podem produzir estresse no indivíduo, quando experienciado de forma intensa (Hocke, 1995). Esse fenômeno é universal, fruto de uma experiência intensa e aflitiva, que parece exercer uma enorme influência no comportamento humano (Lazarus, 1966). Desde 1960, há um reconhecimento do estresse como um fenômeno universal presente em seres humanos e animais (Lazarus, 1966). Para Lazarus e Folkman (1984), o estresse constitui as demandas internas ou externas 
que são avaliadas pelo indivíduo como excedente de seus recursos pessoais. Estes autores propuseram um modelo que identifica dois conceitos centrais: a avaliação cognitiva e o coping. A avaliação cognitiva se refere ao julgamento do evento para verificar se a situação é benigna ou se causará danos ao sujeito. O coping, por sua vez, diz respeito aos constantes e mutáveis esforços cognitivos e comportamentais utilizados pelo indivíduo para lidar com o estresse. Dessa forma, o estresse e o coping consistem em interações e ajustamentos contínuos entre o sujeito e o ambiente.

As concepções propostas por Lipp (2000) são semelhantes às de Lazarus e Folkman (1984). Para Lipp, o estresse é uma reação do organismo frente a situações extremamente difíceis e excitantes. No entanto, Lipp concentrou-se nos componentes psicofisiológicos ao investigar esse fenômeno em adultos e crianças. Lipp e Romano (1987) concluíram que o estresse infantil assemelha-se ao do adulto em vários aspectos. Conforme as autoras, as reações de estresse englobam transformações psicológicas, físicas e químicas no organismo, podendo provir de fontes internas ou externas. As fontes internas estão associadas à maneira pela qual o sujeito reage diante de situações provenientes do cotidiano (e.g., pensamentos e o tipo de personalidade), enquanto as fontes externas referem-se aos fatores ambientais. O grau de estresse experimentado pelo sujeito também está relacionado às mudanças enfrentadas em um determinado período de tempo, ou seja, quanto maior o número de mudanças ocorridas em um período de 12 meses, maior será a probabilidade do desgaste do organismo, devido ao déficit de energia adaptativa (Elkind, 1981; Lucarelli, 2000). O impacto desses estressores na vida das pessoas tem recebido ampla atenção na literatura, especialmente relacionado à presença de doença crônica na família (Bradford, 1997; Factor, Perry, \& Freeman, 1990; Konstantareas, Homatidis, \& Plowright, 1992). Lima, Beria, Tomasi, Conceição e Mari (1996) realizaram um estudo epidemiológico e verificaram que entre os eventos de vida que apresentaram maior índice de prevalência está a ocorrência de doença crônica na família. Apesar de haver na literatura controvérsias sobre a definição de doença crônica, existe um consenso geral de que esta condição é prolongada e que pode proporcionar possíveis conseqüências adversas na vida de um indivíduo (Bradford, 1997). A ausência de concordância nesta terminologia manifesta-se nos métodos empregados nos estudos nesta área, e afeta os índices de prevalência das doenças crônicas. Além disto, interfere no desenvolvimento de uma classificação apropriada, visto que são enfatizados diferentes aspectos (etiologia, severidade ou características da doença) pelos pesquisadores. No entanto, Bradford (1997) criticou esta divisão, afirmando que esta posição enfatiza apenas as diferenças psicológicas existentes nas mais diversas patologias. Apesar de Bradford empregar o termo doença (disease) em seu modelo, acredita-se que esse termo deveria ser substituído por enfermidade (illness), por considerar-se que esta se refere à experiência vivida pelo paciente a partir da doença, que por sua vez, vem a ser o entendimento biomédico da enfermidade (McDaniel, Hepworth, \& Doherty, 1994). Nesse sentido, não há uma linha divisória clara entre problemas orgânicos e mentais. É com base nessa noção que se utilizou um paradigma da Psicologia da Saúde, aplicado aos transtornos do desenvolvimento.

\section{Transtornos do desenvolvimento: estressores em potencial}

Entre os comprometimentos do desenvolvimento infantil que parecem afetar profundamente o sistema familiar situam-se os Transtornos Globais do Desenvolvimento (TGD). Os TGD englobam o Transtorno Autista, Transtorno de Rett, Transtorno Desintegrativo na Infância, Transtorno de Asperger e Transtorno Global sem outra especificação. Os TGD se caracterizam pelo comprometimento grave e global em algumas áreas do desenvolvimento, como por exemplo, habilidades de interação social recíproca, habilidades de comunicação, pela presença de comportamentos, interesses e atividades estereotipadas. $\mathrm{O}$ comprometimento qualitativo que define tais condições representa um desvio acentuado em relação ao nível de desenvolvimento do indivíduo, conforme o Manual de Diagnóstico e Estatística de Distúrbios Mentais (DSM-IV, 1994).

Wing (1996) propôs a utilização da terminologia espectro autista para referir-se a uma vasta gama de patologias (e.g., Síndrome do X Frágil, Síndrome de Rett, Síndrome de Asperger, Síndrome de Landau-Kleffner, entre outras) que têm como característica comum o déficit qualitativo na tríade de comprometimentos (linguagem/comunicação, social e imaginação), no entanto, diferem na sintomatologia, etiologia e prognóstico. $\mathrm{O}$ autismo ${ }^{2}$ é considerado o protótipo desses déficits, sendo conceituado como uma síndrome comportamental que compromete o processo do desenvolvimento infantil e que tem múltiplas etiologias (Gillberg, 1990; Rutter, 1996). As características principais do autismo são o comprometimento da interação social e da comunicação e a presença de comportamentos repetitivos e interesses restritos. Não é de causar surpresa que tais características clínicas tenham um determinado impacto na família. Existem evidências de que essas famílias relatam maiores dificuldades comparadas àquelas em que há membros com Síndrome de Down ou com outros comprometimentos do desenvolvimento (Holroyd \& McArthur, 1976). A maioria dos estudos nessa área focaliza o impacto dos TGD nos pais, ao passo que pouca atenção tem sido dada ao impacto nos irmãos. De acordo com a perspectiva biopsicossocial sistêmica, a família deve ser considerada como uma unidade funcional, na qual o impacto de uma enfermidade repercutirá em cada membro e em todos os relacionamentos familiares (Bradford, 1997; Walsh \& McGoldrick, 1998). A criança com autismo afeta direta e indiretamente o funcionamento dos demais membros na família (Morgan, 1988). O irmão é afetado diretamente, pois precisa adaptar-se e enfrentar os problemas severos apresentados pela criança com autismo. Muitas vezes, observa-se que o irmão cuida, brinca, alimenta, veste, auxilia na higiene e ainda se responsabiliza pela medicação do portador de autismo, na ausência (ou presença) dos pais. Além desses aspectos, os irmãos também apresentam preocupações referentes ao futuro deste indivíduo e ainda temem pela aceitação da condição do seu irmão pela comunidade. Sobretudo, necessitam lidar com as deman- 
das presentes no cotidiano como, por exemplo, o manejo de comportamentos disfuncionais e outros aspectos mais indiretos como a ausência dos pais devido à agenda repleta de freqüentes contatos com profissionais de saúde, e ainda com as alterações nos padrões de relacionamento familiar, resultantes da presença de um membro familiar com necessidades especiais. Preocupados em investigar a natureza e a extensão do impacto dessa situação nos irmãos de indivíduos com autismo, diversos estudos (Breslau, Weitzman, \& Messenger, 1981; Geffken \& Morgan, 1993; Gold, 1993; Mates, 1990) foram conduzidos com o intuito de identificar quais variáveis (e.g., idade, ordem de nascimento, gênero, número de filhos, classe sócio-econômica, etc.) estão associadas à maior vulnerabilidade nos irmãos (Cuskelly, 1999).

Gold (1993) investigou as diferenças existentes entre irmãos de crianças com autismo e irmãos de crianças com desenvolvimento típico nos aspectos referentes à depressão, adaptação social e a realização de tarefas domésticas. Os resultados revelaram a existência de uma tendência dos irmãos das crianças com autismo para apresentarem maiores índices de depressão. No entanto, não foram observadas diferenças significativas quanto ao aspecto familiar (tamanho da família, ordem de nascimento) e adaptação social entre os dois grupos estudados. Contudo, os achados demonstraram que as meninas realizam mais atividades domésticas do que os meninos, principalmente no que se refere aos cuidados prestados à criança com autismo. Verifica-se na literatura uma expectativa de que as meninas realizem mais atividades domésticas do que os meninos, na presença de um membro familiar com necessidades especiais. Portanto, é plausível supor que as meninas apresentem maiores dificuldades de adaptação do que os meninos, devido ao acúmulo de responsabilidades (Mates, 1990; McHale, Sloan, \& Simeonsson, 1986; Morgan, 1988). De qualquer modo, os resultados apontam para a necessidade de chamar a atenção dos pais para o perigo de atribuição excessiva de responsabilidades aos irmãos ou, ao contrário, para a total falta de responsabilidades. Para Bignotto (2000), este fato pode ser uma das possíveis explicações para o estresse infantil, uma vez que essas crianças podem experimentar tensão quanto aos papéis desempenhados. Os demais filhos sentem-se no dever de cuidar e de satisfazer as necessidades da criança afetada, uma vez que esta é sempre tratada como sendo a "caçula" (independente da ordem de nascimento). Esta situação pode tornar-se problemática, especialmente quando os pais possuem grandes expectativas em relação aos demais filhos e não dirigem a eles a mesma atenção voltada à criança com o transtorno, ou seja, ao mesmo tempo em que os pais exigem colaboração dos outros filhos, estão menos disponíveis e atenciosos para com eles (Bradford, 1997; McHale, Simeonsson, \& Sloan, 1984). Esta pressão associada à expectativa dos pais em relação aos outros filhos pode se tornar maior na presença de apenas um irmão com desenvolvimento típico (famílias com dois filhos). Este filho pode sentir-se pressionado a compensar as deficiências da criança com necessidades especiais (McHale et al., 1984).

Por outro lado, outros estudos apontaram a existência do impacto positivo do membro com necessidades especiais no irmão com desenvolvimento típico. McHale e colaboradores (1984) realizaram uma breve revisão sobre o assunto e verificaram que as crianças tendiam a assumir maiores responsabilidades e a manifestar maior idealismo e preocupações humanitárias, referentes aos seus objetivos de vida (Farber, 1963). Cleveland e Miller (1977) constataram que as meninas mais velhas assumiam maiores responsabilidades na família, e tendiam a escolher profissões relacionadas à prática das normas de bem-estar social. Outras evidências (Schreiber \& Feeley, 1965) demonstraram que os irmãos de indivíduos com necessidades especiais apresentavam maior amadurecimento, quando comparado ao grupo controle, e ainda demonstravam mais empatia e tolerância para com outros. Nesta mesma linha de investigação, Kaminsky e Dewey (2001) argumentaram que a presença de um membro familiar com necessidades especiais pode repercutir positivamente nos relacionamentos entre irmãos. Estas autoras verificaram que os irmãos de crianças com autismo e Síndrome de Down relataram sentimentos mais intensos de admiração pelo seu irmão, quando comparados ao grupo controle. Além desses aspectos, verificou-se a existência de menor competitividade e de conflitos entre os irmãos dos indivíduos com necessidades especiais do que entre os irmãos com desenvolvimento típico. No entanto, os relacionamentos dos irmãos das crianças com autismo eram menos íntimos, isto é, menos investidos de carga emocional. Tal fato deve-se às características clínicas do autismo, principalmente o comprometimento na interação social recíproca e nas habilidades de comunicação, que inibem a construção de um relacionamento baseado na troca de sentimentos e de pensamentos. Essas mesmas autoras realizaram uma pesquisa recente (Kaminsky \& Dewey, 2002) e encontraram resultados semelhantes aos de McHale e colaboradores (1986), ao investigar a adaptação psicossocial em irmãos de indivíduos com autismo.

Estes achados apontam para a necessidade de modelos teóricos que ampliem as noções de estresse e coping e contemplem os aspectos envolvidos no processo de adaptação familiar (ou não) a problemas crônicos de saúde em seus membros. Portanto, a eventual presença de estresse em irmãos de portadores de TGD será compreendida a partir de um modelo mais amplo que os tradicionais, isto é, ou cognitivo ou sistêmico, pois busca levar em consideração as transações entre esses processos intra e extra-familiares, especificamente na área da psicologia da saúde.

O meta-modelo de Adaptação Familiar à Doença Crôni$\mathrm{ca}^{3}$ proposto por Bradford (1997) surgiu a partir da experiência clínica do autor com as famílias de crianças com doença crônica e de sua constatação sobre a falta de articulação entre o conhecimento gerado pelas pesquisas e sua aplicação clínica. O foco de interesse do autor é a compreensão de fenômenos envolvidos nas condições potencialmente incapacitantes, sejam elas físicas ou mentais. Dessa forma, os aspectos psicossociais de uma determinada condição não são separados da sua dimensão biológica. O modelo preocupa-se em investigar tanto as peculiaridades de uma doença quanto às similaridades com outras condições, não desprezando os processos de desenvolvimento individuais 
e os do ciclo familiar. Bradford (1997) critica o uso indiscriminado e superficial dos conceitos utilizados em áreas mais amplas do conhecimento (saúde mental, desenvolvimento, personalidade), como, por exemplo, estresse, coping, adaptação, etc., sem a devida articulação com os processos familiares e o contexto de saúde.

Bradford (1997) integrou conceitos da teoria sistêmica (Minuchin, 1990) e de teorias cognitivas (Lazarus \& Folkman, 1984; Moos \& Shaefer, 1984; Pless \& Pinkerton, 1975; Wallender, Feldman, \& Varni, 1989), desenvolvendo um metamodelo sobre adaptação familiar a enfermidades crônicas. Esse autor propôs um modelo ancorado em quatro fatores que foram identificados em suas pesquisas na área da Psicologia da Saúde, como influenciando a adaptação familiar, que são: modos de funcionamento familiar, padrões de comunicação intra e extra-familiar, qualidade dos sistemas de saúde, e crenças sobre saúde. Ressaltou, ainda, a importância de se investigar as características individuais das crianças, os desafios impostos pela doença, as estratégias de coping e a rede de apoio social. Conforme o autor, não se pode afirmar que a presença de uma enfermidade, representa um evento inquestionavelmente adverso para a família. Diante desta situação, a família é confrontada com estressores que podem levar, ou não, ao desenvolvimento de estresse ou depressão, se não forem manejados adequadamente. Enfim, a presença de comprometimentos na saúde de qualquer membro na família demanda uma reorganização familiar, afetando cada um de seus componentes de diferentes modos, ao longo do ciclo vital. Partindo desse pressuposto, o objetivo deste estudo foi o de investigar a presença de indicadores de estresse e a qualidade das relações familiares em irmãos de indivíduo com e sem TGD.

\section{Método}

\section{Participantes}

Foram convidadas 64 crianças e adolescentes para participar do estudo, sendo que dois (um de cada grupo) não aceitaram, totalizando 62 crianças e adolescentes distribuídas em dois grupos, sendo o grupo 1 constituído de 32 irmãos de crianças com Transtornos Globais do Desenvolvimento, associado ou não a causas orgânicas, com idades entre 8 e 18 anos (média $=12,97 ; D P=2,85$ ) e o grupo 2 constituído de 30 irmãos de crianças com desenvolvimento típico, com idades entre 8 e 17 anos (média $=12,37 ; D P=$ 2,20). Em ambos os grupos, metade dos participantes era do sexo feminino e a outra metade do sexo masculino. A inclusão de um grupo controle serviu para controlar o possível efeito da fase do desenvolvimento (pré-adolescência e adolescência) dos participantes sobre o estresse infantil.

Em relação ao tipo de escola verificou-se que a maioria (63\%) dos participantes do grupo 1 freqüentava escola pública, ao passo que isso ocorreu em apenas $33,3 \%$ dos participantes do grupo 2. Quanto a escolaridade, constatou-se uma equivalência nas médias nos dois grupos: 6,47 anos de estudo no grupo $1(D P=3,02)$ e 6,67 anos de estudo no grupo $2(D P=2,06)$. Comparando-se os dados referentes à configuração familiar, observou-se que a grande maioria dos participantes do grupo $1(63 \%)$ e do grupo $2(80 \%)$ residia com a mãe, o pai e os irmãos. Notou-se que o percentual que mora com a mãe, padrasto e irmão foi maior no grupo 1 (19\%), contrastando-se com apenas 3,3\% do grupo 2.

Foram considerados como critérios de inclusão para os participantes do grupo 1: diagnóstico do Transtorno Global do Desenvolvimento do irmão realizado há no mínimo um ano, sendo que o tempo médio de diagnóstico foi de 3 anos. Se uma família tinha vários filhos, foi escolhido, para participar do estudo, o irmão com idade mais próxima do portador de TGD. Foram considerados como critérios de exclusão: a presença de outros fatores estressores, tais como, doença física nos demais membros da família (pais ou outros irmãos), morte de um ente querido (ocorrido em um período inferior a 12 meses) e divórcio dos pais (ocorrido em um período inferior a 12 meses). Conforme Elkind (1981), quanto maior o número de mudanças ocorridas em um período de 12 meses, maior será a probabilidade do desgaste do organismo, devido ao déficit de energia adaptativa. Esses dados foram obtidos através do preenchimento da ficha de dados demográficos e de informações sobre a família.

\section{Instrumentos e materiais}

(a) Termo de consentimento pós-informação. Este documento visou informar os participantes sobre os objetivos e os procedimentos do presente estudo. Somente participaram do estudo, crianças e adolescentes que concordaram em participar e cujos pais assinaram o termo de consentimento pósinformação. Esse documento foi assinado em duas vias. Uma cópia permaneceu com a pesquisadora e a outra cópia com o participante.

(b) Ficha sobre dados demográficos e identificação de estressores ${ }^{4}$. Nesta ficha constaram dados sobre a família, tais como, idade dos membros familiares, escolaridade e profissão dos pais e religião, e questões acerca da presença de outros eventos estressores na família. Esse material foi preenchido pelas crianças e adolescentes, com auxílio da pesquisadora.

(c) Ficha sobre informações sobre o portador de TGD. Esta ficha visou obter dados sobre o diagnóstico do membro com TGD. Através desta ficha foram coletadas informações sobre tempo de diagnóstico, características do portador (comunicação, linguagem, habilidades e autocuidado), além da rede de apoio social da mãe. Essa ficha foi preenchida pela mãe do participante, com o auxílio da pesquisadora.

(d) Escala de Stress Infantil - ESI (Lucarelli \& Lipp, 1999). Objetivou avaliar o estresse infantil, em quatro dimensões ${ }^{5}$ : (1) Física, refere-se às respostas do estresse associadas à dimensão física experimentada pela criança, quando esta está sob o efeito do estresse; (2) Psicológica, observa-se a existência de respostas psicológicas comuns que podem ser experimentados pela criança quando esta está sob o efeito do estresse; (3) Psicológica com Componentes Depressivos, 
refere-se a conteúdos ligados a dimensão psicológica associada a um componente depressivo e (4) Psicofisiológica, refere-se às reações físicas do estresse tendo em comum aspectos psicofisiológicos. Os índices de consistência interna obtida por estas dimensões são respectivamente: 0,$79 ; 0,79$; 0,78 e 0,72 . O instrumento é composto por 35 itens, aos quais os sujeitos respondem, utilizando uma escala Likert de cinco pontos, que vai de zero (nunca sente), a quatro, (sente sempre), referentes ao nível de estresse experienciado pelo indivíduo. A escala demonstrou boas propriedades psicométricas. O índice de consistência interna (alpha de Cronbach) obtido pela escala foi de 0,90 e o coeficiente de correlação de Spearman obtido foi de 0,73 , demonstrando correlação aceitável.

(e) Inventário de Rede de Relações (Network of Relationships Inventory, NRI, versão traduzida, adaptada e validada para o português por Schwertz, 1994): Este instrumento foi utilizado para avaliar a percepção do participante sobre as características das suas relações interpessoais com as pessoas do seu convívio próximo (pai, mãe, irmão). $\mathrm{O}$ instrumento contém 21 itens, redigidos em formato Likert de cinco pontos, que vai de um (pouco ou nada) a cinco (máximo), aos quais os participantes respondem de acordo com a sua percepção do grau de relação interpessoal experimentado com as pessoas de seu convívio próximo. As dimensões da escala são: Companheirismo; Conflito; Satisfação; Revelação Íntima; Cuidado; Afeição e Punição. Nas dimensões punição e conflito, quanto menor o escore, pior o relacionamento com o pai, mãe e o irmão. O participante obtém dois escores no inventário, sendo um escore relacionado à percepção das dimensões relacionais positivas, que são: afeição, cuidado, satisfação, revelação íntima e companheirismo, e outro escore associado à percepção das dimensões relacionais negativas, que são: punição e conflito. O instrumento apresenta boas propriedades psicométricas. No que concerne às dimensões relacionais positivas, o alpha de Cronbach variou de 0,87 a 0,91. Já nas dimensões relacionais negativas o alpha de Cronbach variou de 0,79 a 0,83 .

\section{Procedimentos}

Grupo 1 (irmãos de indivíduos com TGD). As escolas especiais e as clínicas de Porto Alegre foram contatadas por telefone. Por questões de distribuição do nível sócio-econômico, objetivou-se incluir instituições públicas e privadas. Contudo, o critério adotado na seleção de locais foi o de conveniência. A pesquisadora entrou em contato com 16 escolas especiais e clínicas. Desses locais, 12 aceitaram participar do estudo, no entanto, apenas 7 atendiam famílias que preenchiam os critérios de inclusão da amostra. Após o consentimento dos locais, as famílias foram contatadas, inicialmente pelas próprias instituições e posteriormente pela pesquisadora, via telefone. Nessa ocasião foram convidadas a participar do estudo, recebendo informações sobre os objetivos e detalhes do mesmo (local, horário, instrumentos, tempo de aplicação). Nos casos de concordância, foi agendado um encontro com os participantes nas suas respectivas casas. Primeiro, foi solicitado que os pais do participante assinassem o termo de consentimento pós-informação. Em seguida, (sem presença dos pais) o participante respondeu a Ficha sobre Dados Demográficos e Identificação de Estressores, a Escala de Stress Infantil e o Inventário de Rede de Relações. A aplicação dos instrumentos foi realizada individualmente, tendo duração de aproximadamente 1 hora. Foi solicitado que a mãe do participante respondesse a Ficha de Informações Sobre o Portador de TGD. A sequiência de aplicação dos instrumentos foi invertida de participante para participante para minimizar os efeitos de um procedimento sobre o outro.

Grupo 2 (irmãos de indivíduos com desenvolvimento típico). As famílias foram selecionadas em escolas públicas e privadas. O critério de seleção foi o de conveniência e indicação. Primeiramente foi solicitada aos pais dos participantes a assinatura do termo de consentimento pós-informação. Após, os participantes preencheram a Ficha Sobre Dados Demográficos e Identificação de Estressores, a Escala de Estresse Infantil e o Inventário de Rede de Relações, sem a presença dos pais. A aplicação dos instrumentos foi feita pela própria pesquisadora e realizada, individualmente, nas casas dos participantes, tendo duração de aproximadamente 1 hora. A sequiência de aplicações envolve os mesmos procedimentos do Grupo 1.

\section{Resultados}

\section{Escala de Stress Infantil}

Ao analisar os dados da Escala de Stress Infantil (ESI), observamos que o escore médio total obtido pelos participantes dos dois grupos foi de $26,84(D P=14,90)$, portanto muito abaixo do ponto de corte (105). O escore médio total no grupo 1 foi de $26,38(D P=15,14)$ e no grupo 2 de 27,33 $(D P=14,88)$. O teste $t$, para amostras independentes, não detectou diferenças estaticamente significativas entre as médias dos dois grupos $(t=0,118 ; g l=60 ; p=0,90)$. A Tabela 1 informa as médias, desvios-padrão, e escores mínimos e máximos obtidos pelos grupos 1 e 2, em cada uma das dimensões da escala. A comparação dos escores das dimensões do estresse, através do teste $t$ para amostras independentes, não mostrou diferenças estatisticamente significativas entre as médias dos grupos (dimensão Física: $t=0,279$; $g l=60 ; p=0,78$; dimensão Psicológica: $t=0,472 ; g l=60 ; p$ $=0,63 ;$ dimensão Psicológica com Componentes Depressivos: $t=0,256 ; g l=60 ; p=0,79$; e dimensão Psicofisiológica: $t=367 ; g l=60 ; p=0,71)$. Portanto, a análise dos dados da Escala de Stress Infantil não revelou diferenças estatisticamente significativas quanto à presença de indicadores de estresse nos grupos investigados. A partir destes resultados, podemos afirmar que o grupo de irmãos de indivíduos diagnosticados com TGD não apresentou níveis mais altos de indicadores de estresse do que o grupo de irmãos com indivíduos com desenvolvimento típico (DT). 
Tabela 1

Distribuição de médias, desvios padrões e escores mínimo e máximo obtidos da Escala de Stress Infantil nos 2 grupos

\begin{tabular}{lrrrrrc}
\hline \multicolumn{1}{c}{ Dimensões do stress } & \multicolumn{3}{c}{ Grupo 1 $(n=32)$} & \multicolumn{2}{c}{ Grupo 2 $(n=30)$} \\
\cline { 2 - 7 } & $M$ & $D P$ & $\begin{array}{c}\text { Escores } \\
\text { Min - Max }\end{array}$ & $M$ & $D P$ & $\begin{array}{c}\text { Escores } \\
\text { Min - Max }\end{array}$ \\
\hline Físico & 4,44 & 4,32 & $0-17$ & 4,33 & 3,40 & $0-14$ \\
Psicológico & 8,06 & 5,99 & $0-26$ & 9,17 & 5,57 & $0-19$ \\
$\begin{array}{l}\text { Psicofisiológico } \\
\text { Psicológico com componentes } \\
\text { depressivos }\end{array}$ & 7,84 & 4,06 & $2-21$ & 7,33 & 4,09 & $0-21$ \\
\hline Total & 6,16 & 4,57 & $0-16$ & 6,53 & 5,10 & $0-19$ \\
\hline
\end{tabular}

\section{Inventário de Rede de Relações}

Quanto aos resultados do Inventário de Rede de Relações, observou-se a presença de diferenças estatisticamente significativas entre os escores médios da dimensão relacional negativa irmão $(t=3,87 ; g l=60 ; p=0,01)$ e da dimensão relacional positiva pai $(t=2,32 ; g l=55 ; p=0,02)$. Na primeira dimensão, observou-se que o grupo 1 obteve média mais alta do que o grupo 2, indicando a presença de maiores desentendimentos e de opiniões contraditórias entre os irmãos do grupo 1. Na segunda dimensão, verificou-se que o escore do grupo 2 foi mais alto que o grupo 1 , revelando que o grupo 2 demonstra um maior contentamento do seu relacionamento com o pai. O teste $t$ para amostras independentes também mostrou diferenças significativas nas dimensões satisfação pai $(t=3,02 ; g l=55 ; p=0,04)$, satisfação mãe $(t=3,31 ; g l=60 ; p$ $=0,02)$ e cuidado pai $(t=2,54 ; g l=55 ; p=0,01)$ e conflito irmão $(t=4,74 ; g l=60 ; p=0,01)$. Nas dimensões satisfação pai, satisfação mãe, e cuidado pai, observou-se que os escores do grupo 2 foram significativamente mais altos do que os do grupo 1, indicando uma maior satisfação do participante com o seu relacionamento com o pai e a mãe, além de apresentar sentimentos mais intensos de proteção, zelo e ajuda em relação ao pai. Na dimensão conflito irmão os escores do grupo 1 foram significativamente mais baixos que do grupo 2 , demonstrando a existência de maiores conflitos e desentendimentos entre os irmãos do grupo 1 . No que diz respeito às demais dimensões, as Tabelas 2 e 3 apresentam os dados referentes à distribuição dos escores de cada dimensão por grupo.

Tabela 2

Média e desvio padrão dos escores do Inventário de Rede de Relações dos 2 grupos em relação ao Pai e à Mãe

\begin{tabular}{|c|c|c|c|c|c|c|c|c|}
\hline \multirow{3}{*}{ Dimensões } & \multicolumn{4}{|c|}{ Grupo $1(n=32)$} & \multicolumn{4}{|c|}{ Grupo $2(n=30)$} \\
\hline & \multicolumn{2}{|c|}{ Pai } & \multicolumn{2}{|c|}{ Mãe } & \multicolumn{2}{|c|}{ Pai } & \multicolumn{2}{|c|}{ Mãe } \\
\hline & $M$ & $D P$ & $M$ & $D P$ & $M$ & $D P$ & $M$ & $D P$ \\
\hline Companheirismo & 2,71 & 1,07 & 3,30 & 0,97 & 3,09 & 0,96 & 3,56 & 0,88 \\
\hline Conflito $^{6}$ & 2,29 & 0,87 & 2,07 & 0,71 & 1,96 & 0,64 & 2,19 & 0,70 \\
\hline Satisfação & 3,07 & 1,06 & 3,76 & 0,86 & $3,91(*)$ & 1,00 & $4,40(*)$ & 0,61 \\
\hline Revelação íntima & 1,78 & 0,95 & 2,94 & 1,29 & 1,92 & 1,06 & 3,03 & 1,41 \\
\hline Cuidado & 3,12 & 1,06 & 3,67 & 1,00 & $3,79(*)$ & 0,90 & 3,85 & 1,06 \\
\hline Punição & 2,31 & 1,04 & 2,55 & 1,00 & 2,74 & 0,90 & 2,82 & 0,90 \\
\hline Afeição & 4,18 & 0,96 & 4,48 & 0,70 & 4,57 & 0,84 & 4,68 & 0,65 \\
\hline Total positiva & 2,93 & 0,86 & 3,55 & 0,84 & $3,42(*)$ & 0,71 & 3,88 & 0,58 \\
\hline Total negativa & 2,34 & 0,92 & 2,35 & 0,75 & 2,25 & 0,67 & 2,46 & 0,73 \\
\hline
\end{tabular}

(*) $p<0,05$

Tabela 3

Médias e desvios padrões dos escores do Inventário de Rede de Relações dos 2 grupos em relação ao Irmão

\begin{tabular}{llllc}
\hline \multirow{2}{*}{ Dimensões } & \multicolumn{2}{c}{ Grupo 1 $(n=32)$} & \multicolumn{2}{c}{ Grupo 2 $(n=30)$} \\
\cline { 2 - 5 } & $M$ & $D P$ & 3,01 & 0,97 \\
\hline Companheirismo & 3,24 & 1,12 & 2,65 & 1,13 \\
Conflito & $1,57(*)$ & 0,58 & 3,38 & 1,01 \\
Satisfação & 3,81 & 0,97 & 2,10 & 1,24 \\
Revelação íntima & 1,96 & 1,14 & 3,45 & 1,10 \\
Cuidado & 3,90 & 0,95 & 2,13 & 1,17 \\
Punição & 1,60 & 0,92 & 3,81 & 1,02 \\
Afeição & 4,18 & 0,94 & 3,07 & 0,87 \\
\hline Total positiva & 3,35 & 0,90 & 2,50 & 1,00 \\
Total negativa & $1,64\left(^{*}\right)$ & 0,72 & & \\
\hline
\end{tabular}

(*) $p<0,05$ 


\section{Discussão}

A ausência de diferenças entre os grupos investigados quanto aos indicadores de estresse pode ser compreendida a partir de diferentes aspectos. Primeiro, houve uma equivalência nos grupos quanto à idade, escolaridade, configuração familiar, sexo e ausência de outros estressores significativos. Segundo, a ausência de indicadores de estresse nos irmãos pode ser resultante do assessoramento que as famílias, como um todo, vêm recebendo dos locais de atendimento, uma vez que o impacto da síndrome na família pode ser mediado pela rede de apoio social e comunicação com o sistema de saúde (Bradford, 1997; Bristol \& Schopler, 1983; Kaminsky \& Dewey, 2002; Perry et al., 1992). Por último, a ausência de indicadores de estresse também deve ser pensada em termos de limitações metodológicas, como por exemplo, particularidades do instrumento utilizado, tamanho da amostra e nível sócio-econômico da família.

Os achados deste estudo apontam para o fato de que o estresse não pode ser compreendido dentro de uma relação causal e linear, no qual a presença de um evento de vida será, necessariamente um preditor de estresse no indivíduo, sendo necessário adotar uma perspectiva transacional (Bradford, 1997; Lazarus \& Folkman, 1984). Os achados do presente estudo corroboram a revisão dos estudos feita por Lobato (1983), indicando que a presença de um irmão com TGD não constitui, necessariamente, um estressor para o irmão com DT.

O modelo biopsicossocial sistêmico forneceu grande parte das ferramentas básicas para se compreender o impacto dos TGD nos irmãos. As mudanças provocadas pela presença de um filho com TGD parecem colocar os irmãos em risco para desenvolver estresse, mas a intensidade deste fenômeno depende de um conjunto de fatores, que pode mediar o impacto do estresse. De acordo como o modelo, deve-se abordar tanto a totalidade do sistema, quanto um nível particular de cada vez (McDaniel et al., 1994). Nesse sentido, a relação que se estabelece entre os irmãos afeta e é afetada pelos padrões de interação familiar (McHale et al., 1984). Outrossim, a qualidade do relacionamento entre os irmãos pode ser entendida, em seu conjunto, como um reflexo dos padrões de interação familiar. Com isso, percebe-se a necessidade de se adotar uma perspectiva transacional para entender a forma com que a presença de um membro com necessidades especiais repercute nos irmãos com DT (Simeonsson \& McHale, 1981). Dentre os fatores relevantes para compreensão do impacto dos TGD nos irmãos, destaca-se a qualidade do relacionamento familiar.

Em relação aos padrões de interação familiar, os dados do Inventário de Rede de Relações indicaram diferenças estatisticamente significativas entre os grupos investigados. Os irmãos de indivíduos com TGD obtiveram escores mais baixos na dimensão relacional negativa irmão e conflito irmão do que os irmãos de indivíduos com DT, indicando a presença de maiores conflitos entre irmãos no grupo 1, o que não surpreende, dadas as características dos TGD. McDaniel e colaboradores (1994) chamam a atenção para a raiva e a culpa que uma condição incapacitante pode gerar nos irmãos "sadios". Conforme Kaminsky e Dewey (2001), as características clínicas do autismo, como o comprometimento na interação social recíproca e nas habilidades de comunicação, inibem a construção de um relacionamento baseado na troca de sentimentos e de pensamentos. Os resultados das escalas também revelaram diferenças estatisticamente significativas entre os grupos quanto à dimensão relacional positiva pai, satisfação pai, satisfação mãe e cuidado pai. Tais resultados mostram que os irmãos dos indivíduos com DT relataram maior satisfação do seu relacionamento com o pai e a mãe, apresentando sentimentos mais intensos de proteção, zelo e ajuda em relação ao pai, do que os irmãos de indivíduos com TGD. Achados semelhantes foram encontrados por McHale e colaboradores (1986), ao examinarem a qualidade do relacionamento entre irmãos de indivíduos com necessidades especiais. Os autores constataram a existência de uma maior coesão familiar no grupo controle, quando comparados ao grupo dos irmãos das crianças com autismo. Perry McGarvey e Factor (1992) investigaram estresse parental, satisfação conjugal e padrões de funcionamento familiar em pais de meninas com Síndrome de Rett. Os autores concluíram que estes pais, quando comparados a pais de crianças com desenvolvimento típico, estão expostos a mais estressores, devido às demandas impostas pela enfermidade. Sabe-se que diante do diagnóstico de uma enfermidade crônica, a família se depara, freqüentemente, com as suas limitações e com os permanentes cuidados especiais exigidos (McDaniel et al., 1994). Além disso, é necessária a reorganização do sistema familiar, através da construção de uma nova identidade e de objetivos familiares decorrentes desta situação.

Em síntese, os achados deste estudo apontam para a necessidade de se adotar uma perspectiva biopsicossocial sistêmica para compreender o processo adaptativo familiar à enfermidade crônica. A ausência de indicadores de estresse no grupo de irmãos pode ser entendida, entre outros fatores, como fruto dos padrões de interação familiar, os quais são influenciados pelo apoio social, incluindo o sistema de saúde. A inter-relação dos sistemas é mediada pelos padrões de comunicação intra e extrafamiliar, que por sua vez, são diretamente afetados pelo sistema de crenças sobre saúde desenvolvidas pela família. Somado a esses fatores destacam-se as características individuais, as estratégias de coping e os desafios específicos relacionados ao transtorno. Desta forma, para compreender a existência ou não de estresse nos irmãos, deve-se focalizar a qualidade das relações familiares e a forma como se inter-relaciona com esses outros fatores. Por outro lado, algumas limitações do estudo podem ter contribuído para a ausência de estresse. Entre esses fatores citam-se o pequeno tamanho da amostra, possíveis dificuldades em relação a compreensão de alguns itens da escala de Stress e aspectos relacionados à severidade dos sintomas dos irmãos com TGD, os quais não foram controlados nesse estudo. Conforme Facion (J.R., comunicação pessoal, 7 de junho de 2003), é possível que sintomas intensos de comportamentos auto-lesivos, por exemplo, eliciem maior estresse. 
O presente estudo mostrou ainda a necessidade de os sistemas de saúde incluírem os irmãos entre suas prioridades. A rápida resposta à chamada de participação nesse estudo atesta a urgência da criação de espaços desta natureza. Sugere-se que as intervenções com esta população possam explorar o potencial do irmão com DT para auxiliar nos cuidados de forma adequada. Em 1983, Lobato já destacava como benéfica a participação dos irmãos com DT em atividades de grupos de apoio.

\section{Agradecimentos}

As autoras agradecem ao $\mathrm{CNPq}$, pelo suporte financeiro, e às colegas Cristina Neves Hugo, pela cuidadosa leitura do material e a Joceline Fátima Zanchettin, pelo auxílio na análise dos dados.

\section{Referências}

Bignotto, M. (2000). O papel dos pais na prevenção do estresse infantil. Em M Lipp (Org.), Crianças estressadas (pp. 101-122). Campinas: Papirus.

Bosa (2002). Autismo: atuais interpretações para antigas observações. In C. Baptista \& C. Bosa (Orgs.), Autismo e educação (pp. 21-39). Porto Alegre: Artes Médicas.

Bradford, R. (1997). Children, families and chronic disease - psychological models and methods of care. Londres: Routledge.

Breslau, N., Weitzman, M., \& Messenger, K. (1981). Psychological functioning of siblings of disabled children. Pediatrics, 67, 344-353.

Cleveland, D., \& Miller, N. (1977). Attitudes and life commitments of older siblings of mentally retarded adults: An exploratory study. Mental Retardation, $3,38-41$.

Cuskelly, M. (1999). Adjustment of siblings of children with a disability: methodological issues. International Journal for the Advancement of Counselling, 21, 111-124.

Elkind, D. (1981). O direito de ser criança: problemas da criança apressada. São Paulo: Fundo Educativo Brasileiro.

Factor, D., Perry, A., \& Freeman, N. (1990). Brief report: stress, social support, and respite care use in families with autistic children. Journal of Developmental Disorders, 20, 139-147.

Farber, B. (1963). Interaction with retarded siblings and goals of children. Marriage and Family Living, 25, 96-98.

Geffken, J., \& Morgan, S. (1993). Perceived competence and behavioral adjustment of siblings with autism. Journal of Autism and Developmental Disorders, 23, 665-679.

Gillberg, C. (1990). Autism and pervasive developmental disorders. Journal of Child Psychology and Psychiatry, 31, 99-119.

Gold, N. (1993). Depression and social adjustment in siblings of boys with autism. Journal of Autism and Developmental Disorders, 23, 147-163.

Hocke, R. R. (1995). Forty studies that changed Psychology: explorations into the history of psychological research ( $2^{\underline{\underline{a}}}$ ed.). Upper Saddle River, New Jersey: Prentice-Hall.

Holroyd, J., \& McArthur, G. (1976). Mental retardation and stress on the parents: a contrast between Down's Syndrome and childhood autism. American Journal of Mental Deficiency, 80, 431-436.

Kaminsky, L., \& Dewey, D. (2001). Siblings relationships of children with autism. Journal of Autism and Developmental Disorders, 31, 399-410.

Kaminsky, L., \& Dewey, D. (2002). Psychosocial adjustment of children with autism. Journal of Child Psychology and Psychiatry, 43, 225-232.

Konstantareas, M., Homatidis, S., \& Plowright, C. (1992). Assessing resources and stress in parents of severely dysfunctional children through the Clarke modification of Holroyd's Questionnaire on resources and stress. Journal of Autism and Developmental Disorders, 22(2), 217-234.
Lazarus, R. S. (1966). Psychological stress and the coping process. Nova York: McGraw-Hill.

Lazarus, R., \& Folkman, S. (1984). Stress, appraisal and coping. Nova York: Springer.

Lima, M., Beria, J., Tomasi, E., Conceição, A., \& Mari, J. (1996). Stressful life events and minor psychiatric disorders: an estimate of the population attributable fraction in a Brazilian community-based study. Journal of Psychiatry in Medicine, 26, 211-222.

Lipp, M., \& Romano, A. (1987). O estresse infantil. Estudos de Psicologia, $2,42-54$

Lipp, M. (2000). O estresse da criança e suas conseqüências. In M. Lipp (Org.), Crianças estressadas (pp. 13-42). Campinas: Papirus.

Lobato, D. (1983). Siblings of handicapped children: a review. Journal of Autism and Developmental Disorders, 13, 347-364.

Lucarelli, M. (2000). O diagnóstico do estresse infantil. In M. Lipp (Org.), Crianças estressadas (pp. 43-64). Campinas: Papirus.

Lucarelli, M., \& Lipp, M. (1999). Validação do inventário de sintomas de stress infantil - ISS-I. Psicologia: Reflexão e Crítica, 12, 71-88.

Manual de Diagnóstico e Estatística de Distúrbios Mentais (DSM-IV). (1994). ( $4^{\underline{a}}$ ed. $)$. Porto Alegre: Artes Médicas.

Mates, T. (1990). Siblings of autistic children: their adjustment and performance at home and in school. Journal of Autism and Developmental Disorders, $20,545-553$.

McDaniel, S., Hepworth, J., \& Doherty, W. (1994). Terapia familiar médica: em enfoque biopsicossocial com problemas de saúde. Porto Alegre: Artes Médicas.

McHale, S., Simeonsson, R., \& Sloan, J. (1984). Children with handicapped brothers and sisters. In E. Schopler \& G. Mesibov (Orgs.), The effects of autism on the family (pp. 327-342). Nova York: Plenum.

McHale, S., Sloan, J., \& Simeonsson, R. (1986). Sibling relationships of children with autistic, mentally retarded, and nonhandicapped brothers and sisters. Journal of Autism and Developmental Disorders, 16, 399-413.

Minuchin, S. (1990). Famílias: funcionamento e tratamento. Porto Alegre: Artes Médicas.

Moos, R., \& Schaefer, J. (1984). Coping with physical illness, new perspectives. Nova York: Plenum.

Morgan, S. (1988). The autistic child and family functioning: a developmentalfamily systems perspective. Journal of Autism and Developmental Disorders, $18,263-280$.

Perry, A., McGarvey, N., \& Factor, D. (1992). Stress and family functioning in parents of girls with Rett Syndrome. Journal of Autism and Developmental Disorders, 22, 235-248.

Pless, I., \& Pinkerton, P. (1975). Chronic childhood disorder: promoting patterns of adjustment. Londres: Henry Kimpton.

Rutter, M. (1996). Autism research: prospectus and priorities. Journal of Autism and Developmental Disorders, 26, 257-275.

Schimdt, C., \& Bosa, C. (2003). Investigações do impacto do autismo na família: revisão crítica da literatura e a proposta de um novo modelo. Interação em Psicologia, 7, 111-120.

Schreiber, M., \& Feeley, M. (1965). Siblings of the retarded: a guided group experience. Children, 12, 221-225.

Schwertz, A. (1994). Tomada de perspectiva na relação pais-filhos adolescentes. Dissertação de Mestrado não-publicada, Pontifícia Universidade Católica do Rio Grande do Sul, Porto Alegre.

Simeonsson, R., \& McHale, S. (1981). Review: research on handicapped children: siblings relationships. Child: Care, Health and Development, 7, 153-171.

Wallender, J., Feldman, W., \& Varni, J. (1989). Physical and psychosocial adjustment in children with spina bifida. Journal of Pediatric Psychology, 14, 89-102.

Walsh, F., \& McGoldrick, M. (1998). A perda e a família: uma perspectiva sistêmica. In F. Walsh \& M. McGoldrick (Orgs.), Morte na família: sobrevivendo às perdas (pp. 187-198). Porto Alegre: Artes Médicas.

Wing, L. (1996). The autistic spectrum: a guide for parents and for professionals. Londres: Constable. 


\section{Notas}

1 Este trabalho faz parte dos estudos de Mestrado da primeira autora, sob orientação da segunda autora, no Programa de Pós-Graduação em Psicologia do Desenvolvimento da Universidade Federal do Rio Grande do Sul, Porto Alegre, RS.

2 Para mais informações sobre a terminologia utilizada nesta área, sugere-se consultar Bosa (2002).

3 Para mais esclarecimentos sobre o modelo de adaptação familiar à doença crônica, sugere-se consultar Schimdt \& Bosa (2003)

4 A ficha sobre dados demográficos e Identificação de Estressores e a Ficha sobre Informações sobre o Portador de TGD foram especialmente elaborados para este estudo.

5 Alguns dos participantes eram mais velhos do que a amostra utilizada na validação da Escala de Stress Infantil. Apesar dessa limitação, esse instrumento foi utilizado devido à carência de medidas de estresse apropriadas para faixa etária em questão.

6 Nas dimensões conflito e punição, quanto menor o escore, pior o relacionamento com o pai, mãe e o irmão.

Vanessa Fonseca Gomes, mestre em Psicologia do Desenvolvimento pela Universidade Federal do Rio Grande do Sul, é doutoranda no mesmo programa de pós-graduação. Endereço para correspondência: Rua Gal. Couto de Magalhães, 1155, ap. 601 (Higienópolis); Porto Alegre, RS; CEP 90540-131. Tel.: (51) 3343-9117.E-mail: vanessafgomes@yahoo.com

Cleonice Bosa, doutora em Psicologia pelo Instituto de Psiquiatria da Universidade de Londres, é professora no Programa de Pós-graduação em Psicologia do Desenvolvimento da Universidade Federal do Rio Grande do Sul.E-mail: cleobosa@uol.com.br 